\title{
Hyp-1 protein from St John's wort as a PR-10 protein
}

\author{
JOANNA ŚliWiaK ${ }^{1}$, ZBigniew DaUTER ${ }^{2}$, MARIUSZ JASKOLSKI ${ }^{1,3 *}$ \\ ${ }^{1}$ Institute of Bioorganic Chemistry, Polish Academy of Sciences, Poznań, Poland \\ ${ }^{2}$ Synchrotron Radiation Research Section, National Cancer Institute, Argonne National Laboratory, Argonne, USA \\ ${ }^{3}$ Faculty of Chemistry, Adam Mickiewicz University, Poznań, Poland \\ *Corresponding author: mariuszj@amu.edu.pl
}

\begin{abstract}
PR-10 proteins form a large subclass of plant pathogenesis-related proteins that are expressed in response to harmful environmental factors in a wide range of species. Although their function is still not clear, structural data suggest that their characteristic internal hydrophobic cavity can bind relevant plant small-molecule mediators. Hyp-1 from St John's wort (Hypericum perforatum), initially proposed as a catalyst for the biosynthesis of hypericin, was eventually shown to share sequence similarity and a folding pattern with PR-10 proteins. The crystal structure of Hyp-1 in complex with fluorescent probe ANS reveals three distinct and separated binding sites that are unique among PR-10 proteins. The structure can provide guidance in our quest for the true physiological ligands of Hyp- 1 .
\end{abstract}

\section{Introduction}

One of several plant defense mechanisms is based on a number of genes that are expressed in response to harmful abiotic and biotic factors, such as drought, salinity, cold, oxidative stress or pathogens. The pathogenesis-related (PR) proteins encoded by these genes have been divided into 17 classes, based on function, amino acid sequence, and biochemical activity. This classification groups proteins with common function, such as chitinases, defensins, glucanases, peroxidases, proteases, etc. Class 10 (PR-10), however, groups proteins with a well-established canonical fold but without a clearly determined biological function (Fernandes et al., 2013). PR-10 proteins are small ( $\sim 18 \mathrm{kDa})$, mostly cytosolic and have acidic isoelectric point (pI). Their accumulation in roots, seeds and senescent leaves indicates their role in signaling and/or development. Indeed, studies on abscisic acid (ABA) receptors in Arabidopsis thaliana revealed that the cytosolic ABA-binding moiety has a PR-10 fold (Umezawa et al., 2010). Also, other structural studies demonstrate the ability of PR-10 proteins to bind such plant mediators as cytokinins, flavonoids, gibberellins or steroids.

\section{Common fold of PR-10 proteins}

The PR-10 folding canon consists of a seven-stranded antiparallel $\beta$-sheet wrapped around a long and elas-

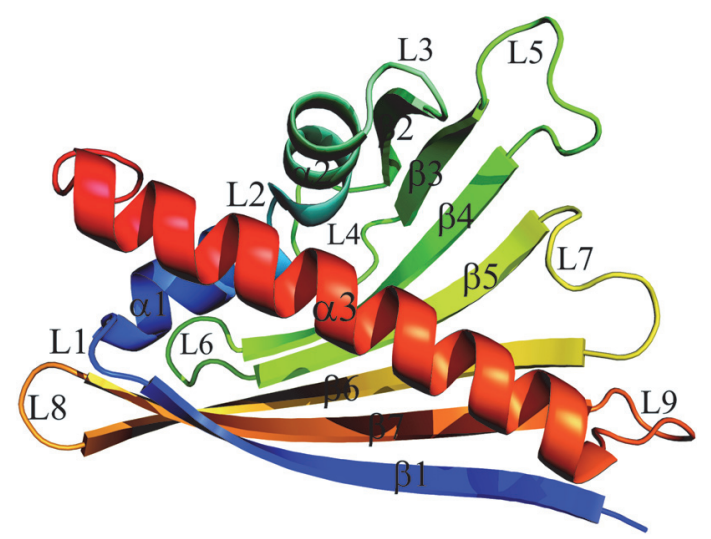

Fig. 1. Secondary structure elements of PR-10 proteins, shown using the example of the Hyp-1 protein (PDB accession code 3IE5; Michalska et al., 2010)

tic C-terminal helix $\alpha 3$, which shows not only conformational but also sequence variability. This "baseball glove" fold creates a large hydrophobic void in the core which is quite unexpected in a rather small globular protein, and suggests a storage function. The hydrophobic cavity has two clearly discernible main entrances: entrance E1 is created by loops and a helix $\alpha 3$, while entrance $\mathrm{E} 2$ is formed between the same $\alpha$-helix and the first strand ( $\beta 1$ ) of the $\beta$-sheet (Fig. 1).

\section{Ligand binding}

The number of NMR and X-ray crystal structures of PR-10 proteins is systematically growing. The structures 


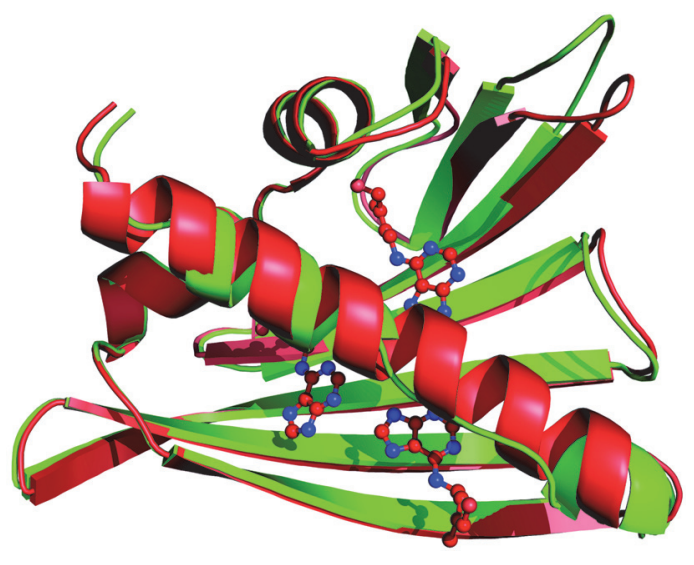

Fig. 2. C $\alpha$ superposition of the yellow lupine LIPR-10.1A protein structure in its free form (green) on the structure from its complex with transzeatin (red) (unpublished). The transzeatin molecules are shown as ball-and-stick models

show the ability of PR-10 proteins to bind variety of physiologically important molecules. In addition, the availability of accurate structures, both in the free form as well as in complexes with ligands, offers a possibility to study the adaptability of the PR-10 fold in binding plant hormones. For instance, the example of the LIPR10.1A protein from Lupinus luteus in its free form (Biesiadka et al., 2002) and in complex with trans-zeatin (unpublished) can be used to explain the binding mode of trans-zeatin (Fig. 2): in the free form the N-terminal part of helix $\alpha 3$ is disordered and the L7 loop is moved to the outside to open entrance E1. Zeatin binding closes this gate and additionally orders the $\alpha 3$ helix.

Analysis of the structures of different PR-10 protein complexes with ligands shows that the interactions with the protein interior are mostly hydrophobic with only sporadic hydrogen bonds formed by hydrophilic side chains, which are rare in the cavity. In such complexes as the Vigna radiata Cytokinin-Specific Binding Protein (VrCSBP) with trans-zeatin (PDB code 2FLH; Pasternak et al., 2006), Medicago truncatula Nodulin 13 (MtN13) with trans-zeatin (4JHG; unpublished) or Phytohormone Binding Protein (PBP) from the same organism with gibberellic acid (3US7; unpublished data) there is only one $\mathrm{E} 1$ entrance to the cavity, which is smaller than in complexes of PR-10 proteins from yellow lupine, such as LIPR-10.1A/zeatin (unpublished) and LIPR-10.2B/zeatin (2QIM; Fernandes et al., 2008) or complexes of the white birch allergen Bet $\mathrm{v} 1$ with deoxycholate, kinetin and naringenin (PDB codes 4A83, 4A85, 4A87; Kofler et al., 2012). In the latter group, the hydrophobic void spans
A

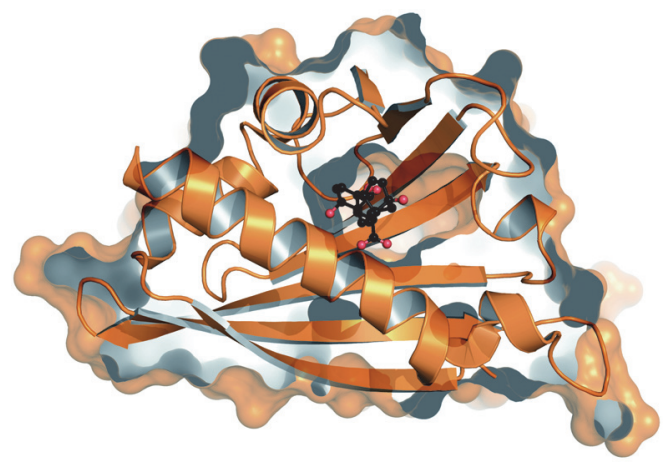

B

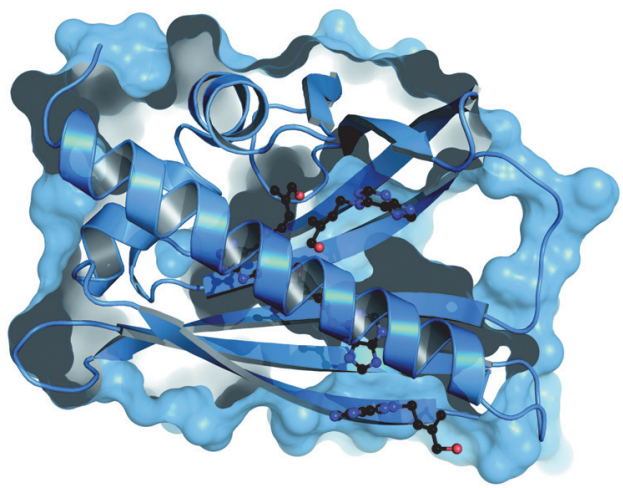

Fig. 3. Two types of shapes of the internal cavity found in PR10 proteins. A) A cavity with only one entrance (E1), illustrated by the structure of the PBP/gibberellic acid complex (PDB accession code 3US7; unpublished). B) A cavity in the form of an elongated hydrophobic tunnel connecting entrances $\mathrm{E} 1$ and $\mathrm{E} 2$, illustrated by the structure of the LIPR-10.2B/trans-zeatin complex (PDB: 2QIM; Fernandes et al., 2008)

the space from E1 to E2 and can accommodate more than two ligand molecules (Fig. 3).

\section{The Hyp-1 protein story}

St John's wort is a millennia-sanctioned medicinal plant, recently rediscovered as a herbal remedy for mildto-moderate depression thanks to the high levels of its pharmacologically active ingredient, hypericin. Hyp-1 had been proposed to catalyze the biosynthesis of hypericin from emodin in vivo (Bais et al., 2003); however, the reaction could not be reproduced (Michalska et al., 2010). Moreover, the highest transcription level of Hyp1 was found in roots, not leaves (Kosuth et al., 2013). Hyp-1, with a molecular mass of $17.8 \mathrm{kDa}$ and $\mathrm{pI}$ of 5.54, shares $\sim 50 \%$ sequence similarity with the PR-10 protein family (Fernandes et al., 2008) and its structural classification in the PR-10 class was confirmed by the crystal structure of Hyp-1 (Michalska et al., 2010), although the root-mean-square deviation for $\mathrm{C} \alpha$ atom superposition on different PR-10 proteins is about $2 \AA$. Co-crystallization 
of Hyp-1 with the purported substrate (emodin) or product (hypericin), as well as calorimetric assays did not confirm their interaction with the protein either.

\section{ANS as a probe of the ligand binding sites of PR-10 proteins}

ANS (8-anilino-1-naphthalene sulfonate) is a fluorescent probe used to investigate hydrophobic ligand-binding sites of macromolecules in ligand-replacement tests coupled with fluorescence measurements. These measurements, which can identify potentially relevant ligands, are based on the ability of ANS to fluoresce (with blue light) in hydrophobic environments (Kundu et al., 2002). The fluorescence is significantly decreased in hydrophilic environments with a bathochromic shift of the fluorescence peak. There are two structures of PR-10 proteins in complex with ANS in the PDB; namely, for proteins from jicama (SPE-16) (PDB: 1TXC; unpublished) and from white birch (Bet v 1) (4A80; Kofler et al., 2012). In the latter case, the structure helped to explain the anomalous ANS displacement data at the molecular level.

\section{Hyp-1 complex with ANS}

Our recently determined crystal structure of Hyp-1 in complex with ANS (unpublished), refined at $2.4 \AA$ resolution, confirms the PR-10 fold of the protein and reveals three principal ligand-binding sites (Fig. 4). Noticeable, even at first glance, is the fact that the binding sites are well separated and defined, quite unlike in the previously characterized ligand complexes of PR-10 proteins. The high specificity of the Hyp- 1 binding sites is unquestionable, as the ANS molecules are observed with highly conserved conformation and orientation in numerous copies of the $28 \mathrm{Hyp}-1$ molecules found in the asymmetric unit (!). The binding site designated as $\mathrm{A} 1$ has its entrance at E1 and the ANS-1 molecule there is stabilized by a hydrogen bond with R27. The binding site designated as A2 has its entrance at E2 and is based on hydrophobic interactions. The third site, designated as $\mathrm{A} 3$, has the shape of a deep pocket formed by invagination of the protein surface, created by tyrosine and lysine residues which bind the ligand mainly through stacking interactions. The superposition of Hyp- 1 from the ANS complex with the free form of the protein does not show any significant changes, particularly of helix $\alpha 3$. This could be explained by the fact that the previously

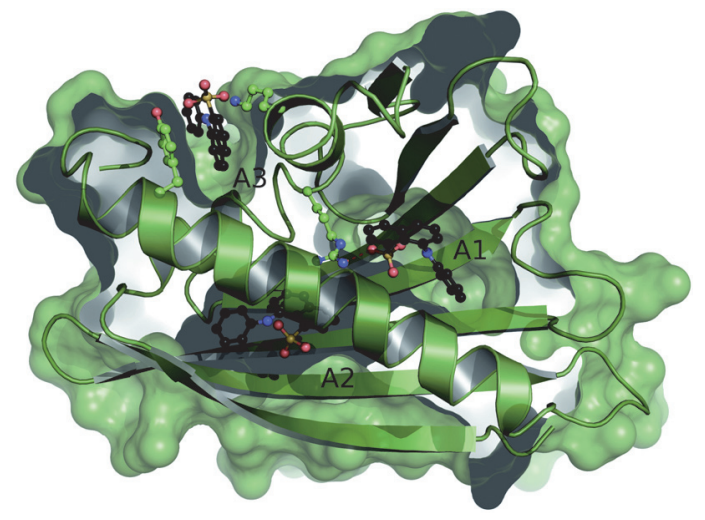

Fig. 4. A crystallographic model of the Hyp-1/ANS complex, illustrating the three separate ligand binding sites of the protein

determined structure of unliganded Hyp-1 (Michalska et al., 2010) has in fact several PEG molecules (from the crystallization buffer) bound non-specifically in the cavity, and this could be sufficient to force the protein to adopt a cargo-bound conformation. Our difficulties with obtaining single crystals of Hyp- 1 , and of PR-10 proteins in general, in a strictly ligand-free form might be interpreted as indicating that in its unliganded form the PR-10 fold has unstable or disordered structural elements. This speculation is corroborated by the case of the LIPR-10.1A protein mentioned above.

\section{Conclusion}

Hyp-1 has been classified as a PR-10 protein both on the basis of a rather high level of sequence similarity and its canonical PR-10 fold. However, the shapes of the ANS binding sites and their evident separation indicate that Hyp- 1 is a rather unique protein in the PR-10 category. The crystal structure of the Hyp-1/ANS complex has revealed three well-defined and unique ligand binding sites. Moreover, the structure provides a valuable atomiclevel basis for a structural interpretation of the fluorescent displacement assays, which can help in identifying the natural ligands of this protein.

\section{Acknowledgments}

Financial support for the project was provided by the European Union within the framework of the European Regional Developmental Fund and by the Polish Ministry of Science and Higher Education (grant No. NN 301 003739).

\section{References}

Bais H., Vepachedu R., Lawrence C., Stermitz F., Vivanco J. (2003) J. Biol. Chem. 278: 32413-32422. 
Biesiadka J., Bujacz G., Sikorski M., Jaskolski M. (2002) J. Mol. Biol. 319: 1223-1234.

Fernandes H., Michalska K., Sikorski M., Jaskolski M. (2013) FEBS J. 280: 1169-1199.

Fernandes H., Konieczna M., Kołodziejczyk R., Bujacz G., Sikorski M., Jaskolski M. (2008) Acta Cryst. F64: 405-408.

Fernandes H., Pasternak O., Bujacz G., Bujacz A., Sikorski M., Jaskolski M. (2008) J. Mol. Biol. 378: 1040-1051.

Kofler S., Asam C., Eckhard U., Wallner M., Ferreira F., Brandstetter H. (2012) J. Mol. Biol. 422: 109-123.

Kosuth J., Jaskolski M., Cellarova E. (2013) Plant Cell Tiss. Org., in press.
Kundu B., Guptasarma P. (2002) Biochem. Biophys. Res. Commun. 293: 572-577.

Michalska K., Fernandes H., Sikorski M., Jaskolski M. (2010) J. Struct. Biol. 169: 161-171.

Pasternak O., Bujacz G., Fujimoto Y., Hashimoto Y., Jelen F., Otlewski J., Sikorski M., Jaskolski M. (2006) Plant Cell. 18: 2622-2634.

Umezawa T., Nakashima K., Miyakawa T., Kuromori T., Tanokura M., Shinozaki K., Yamaguchi-Shinozaki K. (2010) Plant Cell Physiol. 51: 1821-1839. 